\title{
On the determination of local residual stress gradients by the slit milling method
}

\author{
C. Mansilla • D. Martínez-Martínez • \\ V. Ocelík · J. Th. M. De Hosson
}

Received: 12 December 2014 / Accepted: 23 February 2015/Published online: 5 March 2015

(C) The Author(s) 2015. This article is published with open access at Springerlink.com

\begin{abstract}
This paper concentrates on an extension of a rather new methodology to determine local residual gradients at an enhanced lateral resolution using the so-called slit milling technique. The method is based on stress relaxation by making a slit using a focused ion beam and the displacements are measured through digital image correlation so as to calculate the residual stress. Our novel approach consists of a multiple fitting procedure along the length of the slit instead of the commonly applied averaging method. The applicability of our approach is demonstrated when stress gradients exist. In accordance to the Saint-Venant principle in linear elasticity, longer slits are better than shorter slits because of end effects. The proof-of-principle is supported by measurements on steel under controlled bending and by finite element modeling.
\end{abstract}

\section{Introduction}

Residual stresses exist in almost all materials and arise whenever anelastic processes occur, e.g., creep, plastic deformation, surface and thermal treatments, etc. The stresses are mechanically self-equilibrating, i.e., local areas of tensile and compressive stresses sum up to zero forces and moments [1, 2]. Because of its intrinsic self-equilibrating character, the presence of a residual stress state may not be readily apparent. As a consequence, they may be easily overlooked or ignored during engineering

C. Mansilla · D. Martínez-Martínez · V. Ocelík ·

J. Th. M. De Hosson ( $\square)$

Department of Applied Physics, Materials innovation institute

M2i, University of Groningen, Nijenborgh 4,

9747 AG Groningen, The Netherlands

e-mail: j.t.m.de.hosson@rug.nl applications. However, the residual stress state may have beneficial or detrimental effects on material performance [3]. Therefore, a precise knowledge and control of residual stresses are of high practical relevance.

The traditional methods available for measuring residual stress can be divided into destructive and non-destructive. The non-destructive measurement techniques [4] have the advantage of specimen preservation, but they need a "stress-free" reference state which is not always easy to achieve. The most prominent non-destructive method is $\mathrm{X}$-ray diffraction [5], which uses the lattice as strain gage. In contrast, destructive methods are based on measuring the displacements due to stress relaxation [2]. In this case, the residual stresses are "removed" by cutting away the material. A complication of this approach is that the material under stress is partially destroyed and measurements must therefore be made on the remaining material requiring specialized detection methods [6,7]. Various relaxation methods for measuring residual stresses were developed for both general and specific kinds of specimens $[2,8]$. The most commonly used are Hole Drilling and Ring Coring [8], Deep Hole Drilling [9], and the Slit Milling method $[10,11]$. They differ in the specimen geometry, the cutting procedure, and spatial resolution [2].

Recent research focused on downscaling of stress relaxation measurement techniques to a microscale [12-26]. In particular, the residual stresses were relaxed by material removal, typically made using a focused ion beam (FIB) inside a scanning electron microscope (SEM) chamber. Digital image correlation (DIC) is used to find the induced displacements near the slit of the removed material making images before and after the removal $[27,28]$. These recent developments make applications at the local microstructural level feasible.

When a planar slit is introduced into a material, an inplane component of the residual stress can be obtained with 
the slit milling method [24, 29, 30]. However, it provides only the residual stress component normal to the surface of the cut [2]. In this work, a new approach is presented that leads to an improvement of the lateral resolution of the method. This approach we called as "multiple fitting" will make residual stress measurements possible, not only at the microscale but also at the nano-scale. This modification also allows the technique to have access to stress gradients near heterogeneous interfaces. Finite element modeling (FEM) is used in materials with homogeneous and inhomogeneous stress fields, and the theoretical analysis supports the proof-of-concept of this technique.

\section{Experimental details}

Slit milling method

Figure 1 summarizes the procedure that was followed. It combines the ion milling and imaging techniques through a dual beam microscope with DIC software. The residual stresses released in the vicinity of a slit are deduced from the local displacements measured by DIC. The procedure starts with the acquisition of a first SEM image. After capturing the image, a slit is milled by FIB. It is worth mentioning that in general the electron and the ion columns inside a dual beam system have different orientations, which implies that the sample under investigation has to be repositioned for milling. Afterwards, the sample is tilted backwards and a second image of the same area is taken. From the comparison of these two SEM images recorded before and after stress release, the displacement field perpendicular to the plane of the slit is obtained by DIC. Points very close to the slit $\left(|y| / a_{\mathrm{f}}<1, a_{\mathrm{f}}\right.$ represents the depth of the slit) are usually not included in the evaluation because (1) they are often affected by re-deposition during ion milling and (2) their displacements are influenced by the width of a slit in comparison with a crack [29]. Regions of 4-5 times the depth of the slit $\left(|y|<5 a_{\mathrm{f}}\right)$ are considered for the displacements analysis outside the slit. DIC displacements are presented in color, i.e., red and blue colors indicate displacements to the bottom or to the top, respectively. The scale bar of the displacement images lies in the range of tens of nanometers.

The magnitude of the residual stress in the direction perpendicular to the plane of the slit, $\sigma_{\mathrm{dir}}$, is obtained by comparing the experimentally observed displacements and the values of the displacements obtained from the analytical solution for an infinite length slit $(L \rightarrow \infty)$ in an isotropic linear elastic material [15, 24, 31]:
Fig. 1 Steps involved in the measurement of residual stress by slit milling using a combination of SEM imaging, FIB milling, and DIC image analysis

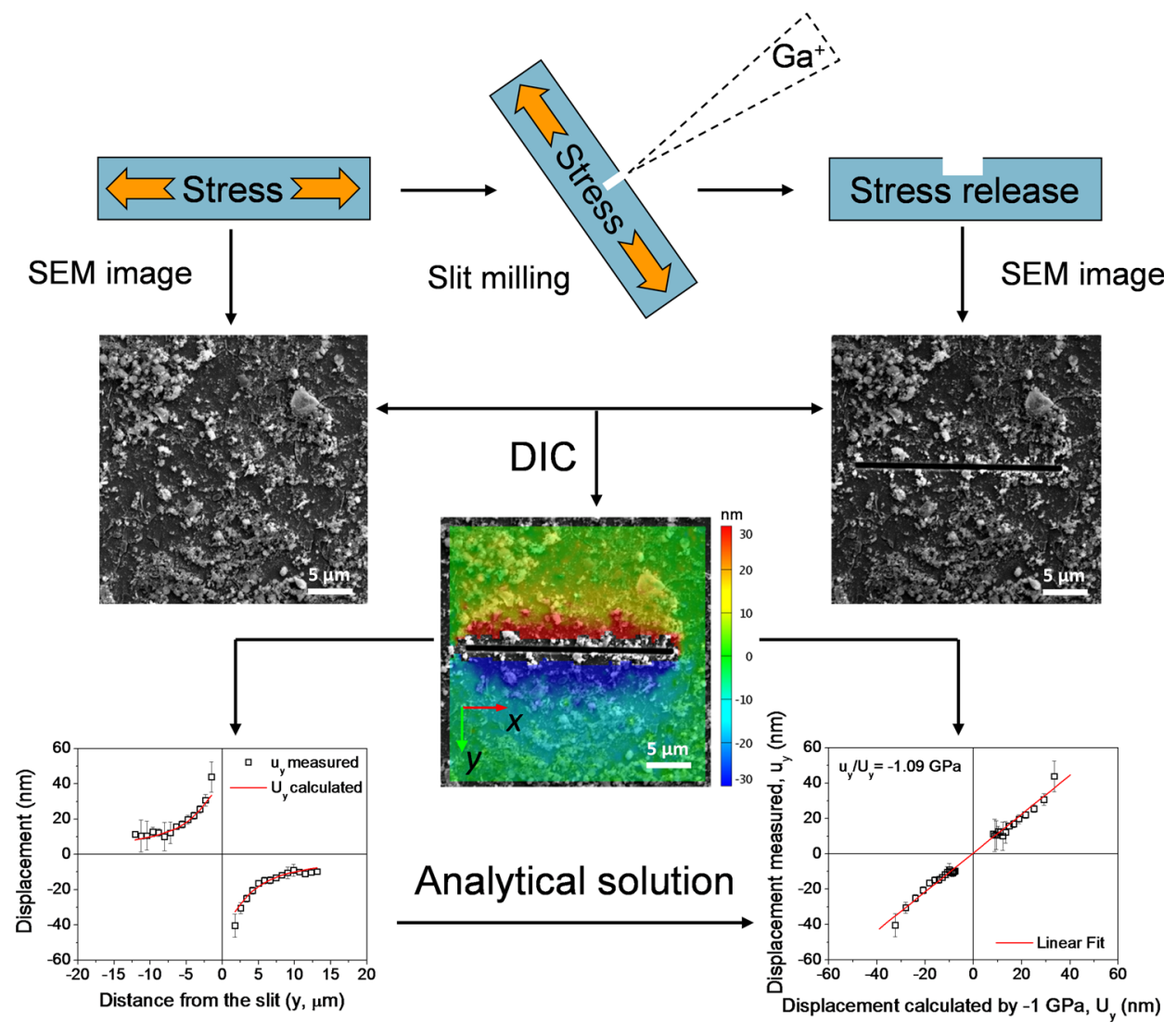




$$
\begin{aligned}
U_{\text {dir }}(d)= & \frac{2.243}{E^{\prime}} \sigma_{\operatorname{dir}} \int_{0}^{a_{\mathrm{f}}} \cos \theta\left(1+\frac{\sin ^{2} \theta}{2(1-v)}\right) \\
& \times(1.12+0.18 \cdot \operatorname{sech}(\tan \theta)) \mathrm{da}
\end{aligned}
$$

where $a_{\mathrm{f}}$ is the depth of the slit, $E^{\prime}=E /\left(1-v^{2}\right), E$ is the Young's modulus, $v$ is the Poisson's ratio, $\theta=\arctan (d / a)$, with $d$ the distance to the slit, and $a$ changing between 0 and $a_{\mathrm{f}}$. The displacements caused by the stress release depend on the slit depth $a_{\mathrm{f}}$ and are directly proportional to the $\sigma / E^{\prime}$ ratio. The extraction of the value of the residual stress requires knowledge of the elastic properties (i.e., Young's modulus and Poisson's ratio). So far, when applying Eq. 1, the measured displacements were averaged over the distance to the slit in a vicinity of the center of the slit (i.e., only one set of data is analyzed). The size of the area in which the displacement field is expected to be uniform and independent of the position along the slit direction in the case of a homogeneous internal stress is often discussed in literature [24, 29]. In our experiments, we use a distance $|x|<0.3 L$. However, the size will be discussed later in relation to longer slits, i.e., $L>10 a_{\mathrm{f}}$.

Scanning electron microscopy and ion milling were carried out in a dual beam microscope (FIB/FEG-SEM) Lyra from Tescan. The electron gun was operated with a voltage of $10 \mathrm{kV}$, constant beam current of $1 \mathrm{nA}$, and a working distance of $9 \mathrm{~mm}$. Image resolution was $768 \times 768$ pixels. The focused ion beam (FIB) was operated with a voltage of $30 \mathrm{kV}$ with a probe current $200 \mathrm{pA}$. DIC analysis of the images was carried out with Aramis 5.3 DIC software [32] using a facet size of $21 \times 21$ pixels with a step between the center of neighbour facets of 11 pixels. The DIC calculation provides the vector of displacement between two tested images for the center of each facet. Since DIC is an image-based method, yttria-stabilized zirconia (YSZ) nanoparticles are typically deposited on the top of the specimen under analysis to enhance the contrast on the surface for optimum results [13, 33, 34]. Depth of the slits was measured after DIC experiment by SEM [24].

\section{Numerical calculations}

FEM has been employed to calculate the displacements generated as a result of the slit milling by FIB. Comsol v. 4.3.0.151 has been used for these calculations. Figure $2 \mathrm{a}$ shows the 3D geometry of the slit used in our experiments including its dimensions: a length $L$, a width $w$, and a depth $a_{\mathrm{f}}$. The origin is at the center of the slit. An initial uniaxial compression stress field in the $y$ direction, $\sigma_{y}$, is considered. The evaluated displacements of the surface, $u_{y}$, are normal to the plane of the slit. Figure $2 b$ shows an example of the surface displacement field $u_{y}$ in the region near the slit, caused by a constant uniaxial stress field $\sigma_{y}=-1 \mathrm{GPa}$, considering a rectangular slit $\left(a_{\mathrm{f}}=2.7 \mu \mathrm{m}, w=0.5 \mu \mathrm{m}\right.$, and $L=23 \mu \mathrm{m})$. Due to the symmetry of the problem, only half of the body was modeled. A rectangular slit is considered instead of a V-shaped one, since the shape of the slit does not have a significant effect on the resulting displacement field [24]. The number of nodes in the model is 51489, and the material was assumed to be linear isotropic with a Young's modulus $200 \mathrm{GPa}$ and Poisson's ratio of 0.24 . As expected, due to the compressive stress applied, the displacements have negative values indicating closure of the slit. Moreover, the displacement field is symmetric from the center of the slit, due to the presence of a constant stress field. As expected, the displacements decrease from the center of the slit to the edges.

\section{Experimental material}

Phytime maraging steel material was used in our experiments. Grains sizes were determined by orientation imaging microscopy performed on Philips XL30 FEG scanning electron microscope equipped with TSL OIM system based on DigiView 3 camera. Internal stress gradient near the sample surface was introduced by gaseous nitriding in the range of $450-500{ }^{\circ} \mathrm{C}$.

\section{Results and discussion}

When the slit milling method is used, the calculations are done assuming the presence of constant stress through the plane of the slit and using the analytical solution for an isotropic linear elastic material with crack (Eq. 1). This approach works very well when homogenous displacements are detected by DIC (all points experience the same displacements), and an averaging procedure over the central part of the slit is used to reduce the noise of the measurement. However, when DIC images with non-homogeneous displacements (each point was subjected to a different value of stress/strain) are detected, a loss of resolution occurs during the averaging procedure. Therefore, we need a new approach that we called 'multiple fitting'. In this approach, displacement field along each row of facets used in DIC analysis is considered and analyzed separately. It allows enhancing of the lateral resolution.

Figure 3 compares both situations observed experimentally after DIC analyses. The length of the slit in both cases is $12 \mu \mathrm{m}$. The second DIC image (Fig. 3b) shows varying displacements through the length of the slit, in contrast to the situation where displacements are homogeneous in the central part of the slit (Fig. 3a). The value of the stress that would be obtained after averaging the displacements in both cases is depicted in Fig. $3 \mathrm{c}$ and d, respectively (solid square symbols). In addition, the values of residual stress obtained using our multiple fitting 

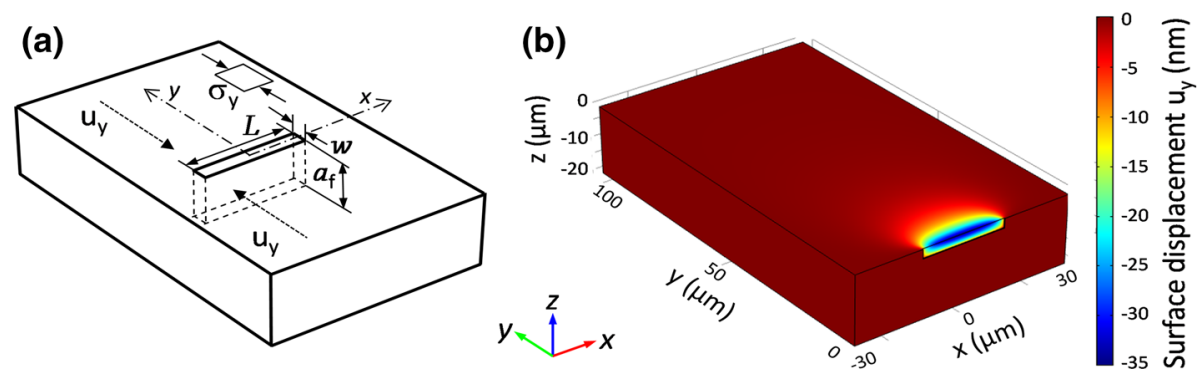

Fig. 2 a Scheme of the 3D geometry of the slit and its dimensions: length $L$, width $w$ and depth $a_{\mathrm{f}}$. An initial uniaxial compressive stress field in $y,-\sigma_{y}$, is considered. The evaluated displacements of the surface, $u_{y}$, are normal to the plane of the slit. The origin of coordinates is placed at the center of the slit. b Example of the surface displacement field $u_{y}$ in the region near the slit, caused by a constant uniaxial stress field $\sigma_{y}=-1 \mathrm{GPa}$. Slit dimensions: $23 \mu \mathrm{m}$ length, $0.5 \mu \mathrm{m}$ width, and $2.7 \mu \mathrm{m}$ depth
Fig. 3 Homogeneous (a) and non-homogeneous (b) surface displacements fields measured by DIC after stress release. Residual stress values obtained using averaging (solid symbols) and multiple fitting (hollow symbols) approaches for both cases are presented in $\mathbf{c}$ and $\mathbf{d}$, respectively. The overall errors of the fittings calculated using Eq. 2 are also represented (see Fig. 4 for details)
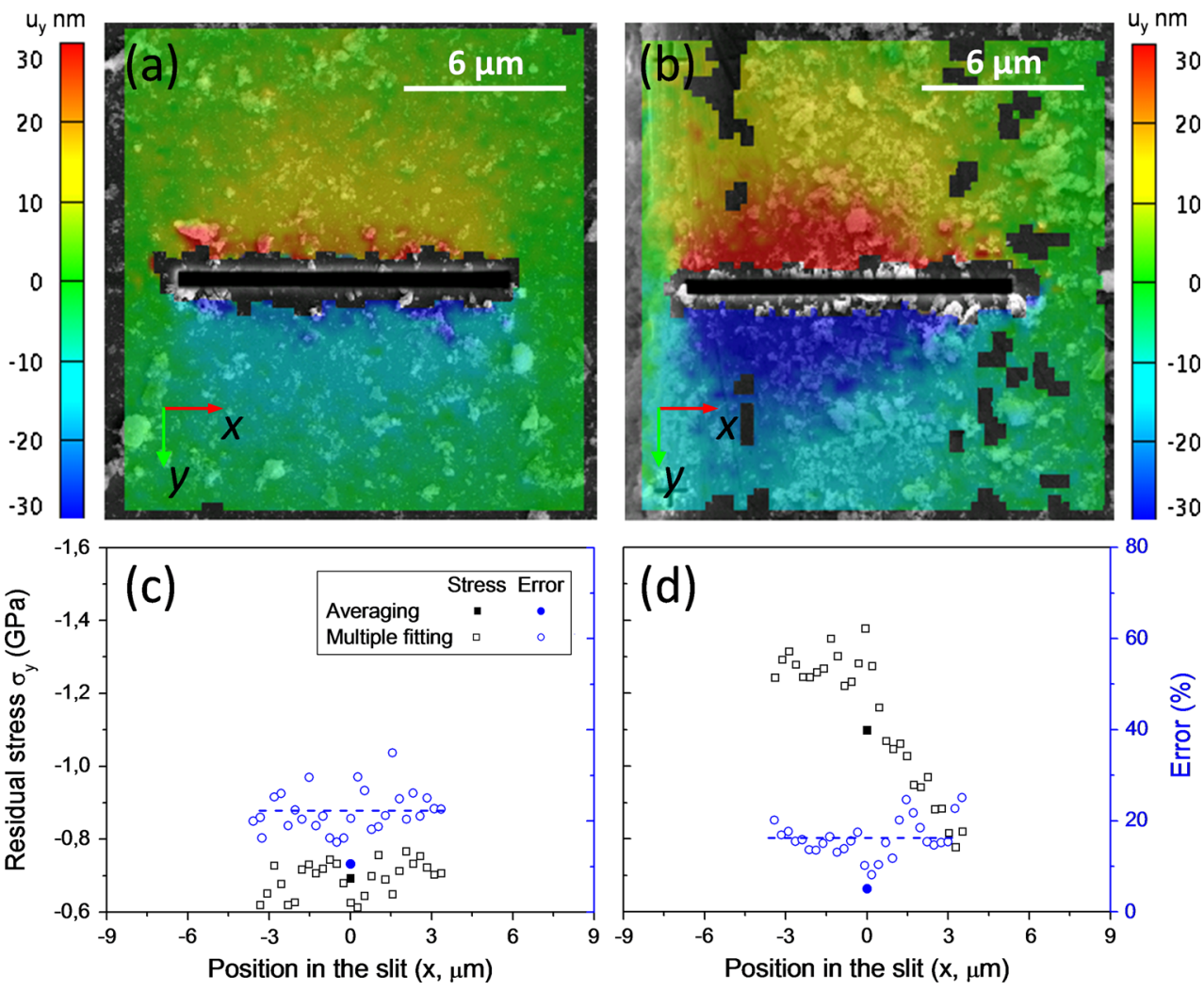

approach (one value for each set of displacements perpendicular to the slit) are also presented (hollow square symbols). A clear stress gradient is observed in Fig. 3d, while Fig. 3c shows a constant value of stress in that region. The differences in the value of stress obtained with both methods, i.e., with averaging (solid symbols) vs. without (hollow), indicate that the protocol of calculation must be different in each case. In the case of homogenous displacements, the value obtained after averaging lies in the middle and averaging is demonstrated to be correct. In the second case, the average does not reflect the real stress state and a multiple fitting has to be done through the length of the slit in order to avoid unrealistic answers and loss of information.

Our approach has the clear advantage of increasing the lateral resolution of the slit milling method, although the procedure has the disadvantage of not performing an averaging process of the displacements measured by DIC. Figure $4 \mathrm{a}$ and $\mathrm{b}$ shows the displacement profiles after averaging for both situations depicted in Fig. 3, while Fig. 4c and $\mathrm{d}$ shows representative profiles during the multiple fitting approach. Note that the points in the former plots show error bars, which are the result of the averaging of the DIC data, i.e., the standard deviation of the displacements. 
Fig. 4 Plots of displacements versus distance to the slit obtained from the fields shown in Fig. 3 (left homogeneous, right non-homogeneous). Top after averaging (error bars represent the standard deviation). Bottom representative sets of data of the multiple fitting analysis. In all cases, the fittings according to Eq. 1 are shown as red lines. The errors calculated using Eq. 2 are depicted on the bottom of each graph. Blue lines represent the fitting curves plus and minus the overall errors (Color figure online)
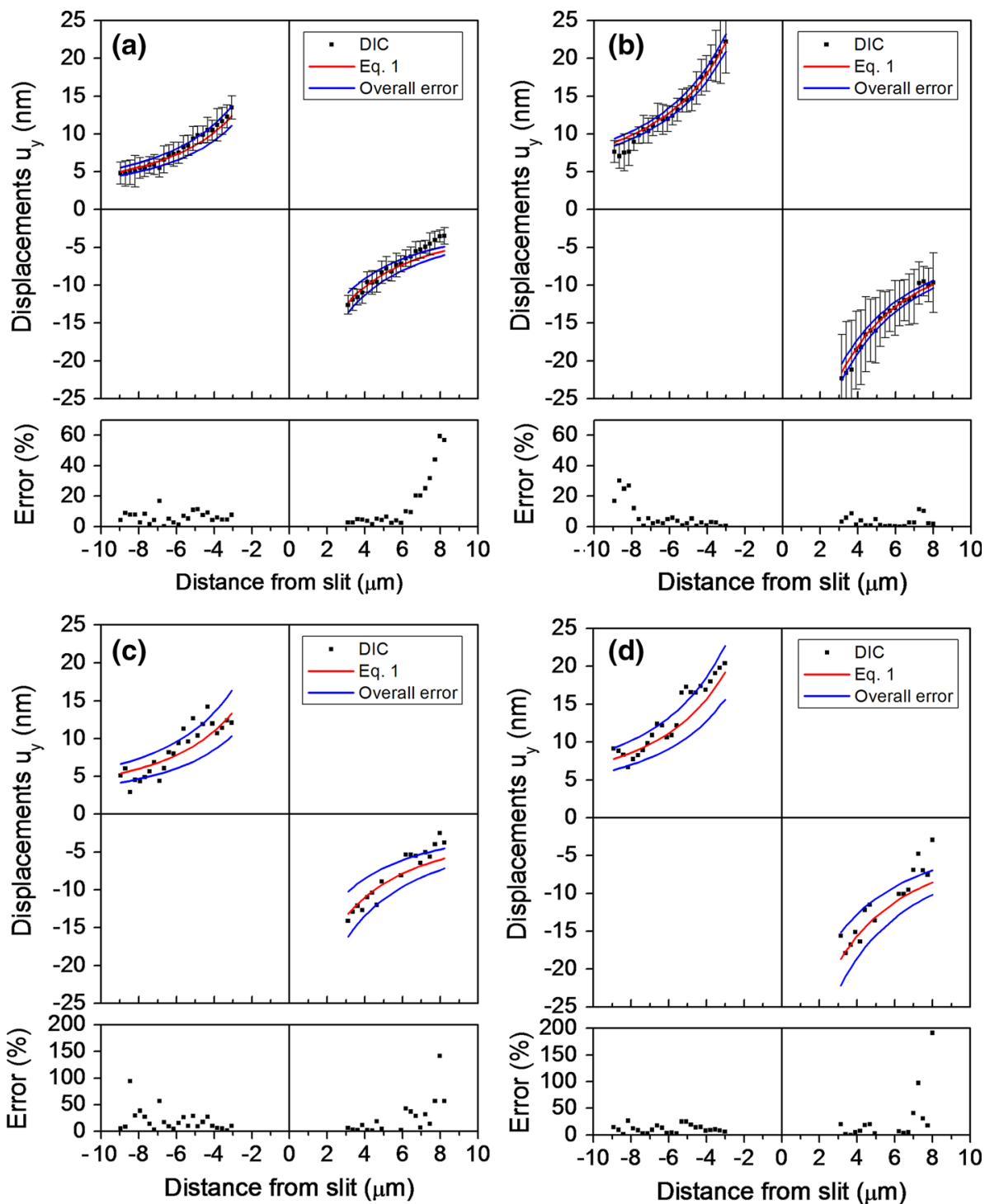

As expected, the error bars are much smaller in the case of a homogeneous displacement field (Fig. 4a). The red colored lines in Fig. 4 represent the best fitting curve (Eq. 1) for all cases. An estimation of relative error of the fitting can be calculated using the following expression:

$$
\begin{aligned}
& \operatorname{Error}(\%) \\
& =100 \times\left|\frac{\text { Displacement (DIC) }- \text { Displacement (curve) }}{\text { Displacement (DIC) }}\right|
\end{aligned}
$$

These errors are calculated for each point in the plots, and are represented in the bottom part of Fig. $4 a-d$. The values are higher in the curves obtained after multiple fitting, indicating a certain loss of precision in the calculation. However, it can be noted that some points may deviate from the general trend even after averaging (cf. points in Fig. 4a, whose relative errors are close to $70 \%)$. The overall error for a given fit can be expressed as the average of all the values calculated using Eq. 2. These errors are depicted in Fig. 4 as curves colored blue, which are the fitted curve (Eq. 1) plus and minus the overall error. These errors are also depicted in Fig. $3 \mathrm{c}$ and $\mathrm{d}$ for all the fittings. First, it can be seen that the distribution of errors is random through the length of the slits. This is particularly relevant in case of the non-homogeneous situation, since very different values of stress are measured. Second, it can be observed that the overall errors for the averaging procedures (10.6 and $5.1 \%$ for homogeneous and non-homogeneous case, respectively) are lower than the errors observed during the multiple fittings (average values of 22.2 and $16.2 \%$ for homogeneous and non-homogeneous case, respectively, which are represented by dashed blue 
Fig. 5 Displacement fields in $y$ direction obtained by FEM in the presence of uniaxial constant (a) and non-constant (b) stress fields. In case of constant stress, a residual stress of $-1 \mathrm{GPa}$ was used. In case of non-constant, a linear variation in $x$ from -1.58 to $-0.43 \mathrm{GPa}$ within the length of the slit $(23 \mu \mathrm{m})$ was employed. The corresponding displacement profiles are shown in $\mathbf{c}$ and d. Each color corresponds to a value of $x$, with $x=0$ representing the center of the slit (Color figure online)
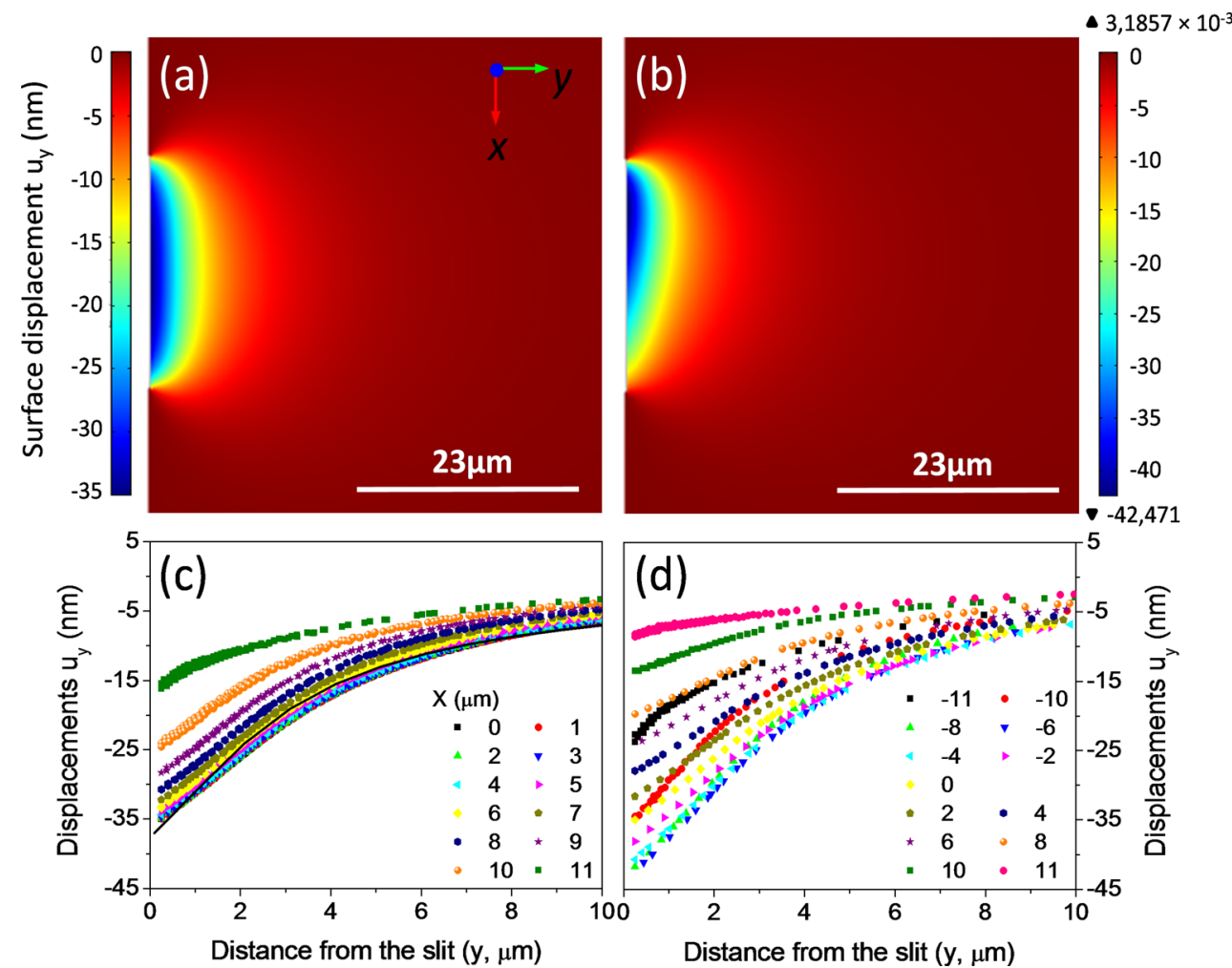

lines in Fig. 3c, d). This difference indicates the loss of accuracy due to the multiple fitting approach. It is worth mentioning that the data represented in Fig. 4c and d were selected because the overall errors of those fits were close to the average of the overall errors of both multiple fittings (in other words, the corresponding points in Fig. $3 \mathrm{c}$ and $\mathrm{d}$ were close to the dashed line). Therefore, the larger separations of the blue lines in the plots after multiple fitting (Fig. 4c, d) when compared with the respective plots after averaging (Fig. 4a, b) represent a valid quantitative picture of the loss of precision when using the multiple fitting approach. Possibly, the errors in multiple fitting could be reduced by averaging of the DIC displacements within the few closest columns of displacements.

It is clear that the averaging approach should not be used if a non-homogeneous displacement field exists, although it is valid for homogeneous ones. In such a situation, the multiple fitting approach reveals the variation of stress along the length of the slit, although the fitting error is larger due to the lack of averaging.

FEM simulations were carried out to validate the applicability of Eq. 1 in case of a non-homogeneous stress. Figure 5 compares the surface displacement fields in the $y$ direction obtained after simulation in the presence of a constant (a) and a linear (b) stress field. In the former case, a value of $-1 \mathrm{GPa}$ is used, while in the latter a linear variation of stress occurs along the $x$ edge of the simulation body (ca. from -1.58 to $-0.43 \mathrm{GPa}$ along the $23 \mu \mathrm{m}$ length slit). The center of the slit is located at $x=y=0.0$ in both cases. The function used was $\sigma_{\mathrm{y}}=50(\mathrm{MPa} / \mu \mathrm{m}) \cdot x(\mu \mathrm{m})-1(\mathrm{GPa})$.

These two simulations resemble the results obtained by DIC (see Fig. 3). The value of the displacements obtained in the middle of the length of the slit is comparable in both cases, as expected (stress is -1 GPa in the center of the slit in both cases). It can be seen that the maximum value of displacements is of the order of 30-40 nm, being comparable to values obtained by DIC. The corresponding displacement profiles are depicted in Fig. 5c and d. Each profile (i.e., color) corresponds to a position between two ends of the slit (i.e., a particular value of $x$ between $x=-$ 11.5 and $x=11.5 \mu \mathrm{m}$, being the center at $0.0 \mu \mathrm{m})$. All profiles show similar shapes, with displacements decreasing with $y$. In Fig. 5c, where displacements are symmetric with respect to the center of the slit, only half of the slit is shown and positive values in $x$ are considered. It can be seen that the maximum displacement is located along horizontal line at the center $(x=0 \mu \mathrm{m})$ and decreases to the edge $(x=11.5 \mu \mathrm{m})$.

Nevertheless, from a distance of $6 \mu \mathrm{m}$ from the center of the slit, the simulated shapes and values start to deviate and are not in accordance with the analytical solution, shown in Fig. $5 c$ by the solid line. In Fig. 5d, the profiles extracted from Fig. $5 b$ are depicted. Since there is no symmetry in the $x$ axis, profiles at different distances from the center of the slit are displayed. From -11 to $-8 \mu \mathrm{m}$, the displacements observed increase due to decreasing separation to the 
edge of the slit. Then, the curves are very similar at $x=$ $-6 \mu \mathrm{m}$ and $x=-4 \mu \mathrm{m}$, and the displacements start to decrease again, with more or less constant steps up to $x=8 \mu \mathrm{m}$. At $x=10 \mu \mathrm{m}$ and $x=11 \mu \mathrm{m}$, the displacements decrease again, but the drop is more pronounced. Thus, it is clear that both parameters (residual stress and proximity to the edges) influence the observed results.

To examine the validity of the multiple fitting approach in both cases, the residual stresses are calculated using Eq. 1 from the profiles depicted in Figs. 5c and d, and compared with the values of stress used in the simulations. In other words, the 'multiple fitting' approach has been employed using the profiles of displacements obtained from the simulations instead of the DIC images that were obtained experimentally. Figure 6 includes those values of residual stress at different distances from the center of the slit, when homogenous (symbols in blue color) and nonhomogeneous (symbols in red color) displacements are considered. Input values of residual stress used in FEM for each case are also included (solid lines). As expected for a homogeneous displacements field, the values of residual stress are symmetric, decreasing from the center of the slit to the edge following a non-linear trend. If these values (symbols in blue color) are compared with the input theoretical values (blue line), we can see that these agree up to an absolute value of $\pm 6.5 \mu \mathrm{m}$, i.e., the $60 \%$ of the length of the slit. However, near the ends of the slit, a strong deviation is observed due to failure of the model employed (which assumes an infinitely long slit). Similar agreement is found in the case of non-homogeneous displacements, where calculated values (symbols in red color) agree with the theoretical input values in the same range of $60 \%$ of the length of the slit. However, stresses are not symmetric

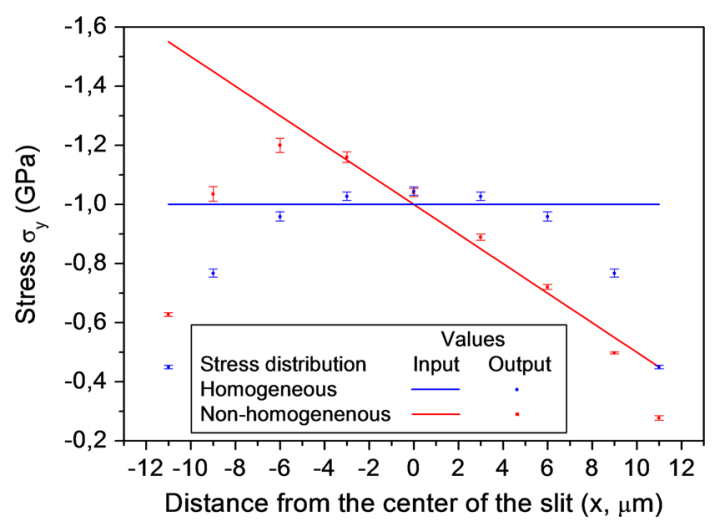

Fig. 6 Values of residual stress (outputs) at different distances from the center of the slit calculated using Eq. 1 on the profiles shown in Fig. 5c and d. Error bars represent the error of the fitting slope (see Fig. 1). Theoretical values of stresses used as input in the FEM calculation are included for comparison (Color figure online) and the deviation occurs in a different way at both edges (as expected from the observed displacements in Fig. 5d). In conclusion, a range of ca. $60 \%$ can be used for short slits with length of around $\sim 20 \mu \mathrm{m}$. In that range, the multiple fitting approach has been shown essential (cf. Fig. 5b). This result also indicates that a selection of a range for averaging is needed even in the case of homogeneous displacements (60\% of the slit is correct in this case).

However, the magnitude of the range of validity of the analytical solution has to be evaluated for other slit lengths. It is expected that end effects due to the finite length of the slit will become relatively less important and that a greater portion of the length slit can be used for a consistent determination of the residual stress. The Saint-Venant principle in the theory of elasticity states that a system of forces in equilibrium applied to a segment of a solid body produces stresses that rapidly diminish with increasing distance from the segment. According to this principle, one would expect that a ten-times larger slit length will diminish the influence of the end effects substantially. Figure 7 shows the surface displacement map in $y$ direction obtained after simulation in the presence of constant (a) and non-constant (b) stress fields for slits ten times longer $(230 \mu \mathrm{m})$ than those shown in Fig. 5. A constant stress of $-1 \mathrm{GPa}$ was considered in Fig. 7a in order to obtain a homogeneous displacements field. Also, a non-constant stress changing linearly from compressive to tensile $(-0.82$ to $0.82 \mathrm{GPa}$ ) through the length of the slit was applied in the second case (Fig. 7b). As before, the center of the slit is located at 0.0 in both cases. The function used was $\sigma_{\mathrm{y}}(\mathrm{MPa})=7.13 \cdot x$, with $x$ in micrometers.

In contrast to the situation where the displacements are homogeneous and negative through the length of the slit (see Fig. 7a), a gradient is observed in Fig. 7b, where displacements change from negative to positive values (from bottom to top part) being 0 at the center of the slit. The corresponding displacement profiles near both long slits are shown in Fig. 7c and d. As before, all profiles show similar shapes, with displacements decreasing with $y$ (regardless of positive or negative value). In case of Fig. 7c, all curves in the central region (i.e., between $\mathrm{x}=0$ and $\mathrm{x}=100 \mu \mathrm{m}$ ) are very similar, and just those showing maximum and minimum values of displacement are shown. Both lines agree quite well with the predictions of Eq. 1 (also included as solid line). However, at larger values of $x$, the curves start to deviate, and the quality of the fittings to Eq. 1 gets worse (result not shown). Therefore, for this slit length, Eq. 1 is applicable in the $87 \%$ of the slit (i.e., the central $200 \mu \mathrm{m}$ of total $230 \mu \mathrm{m}$ ). This result indicates that the useful region is larger for longer slits and therefore longer slits are preferred. Figure $7 d$ shows the collection of 

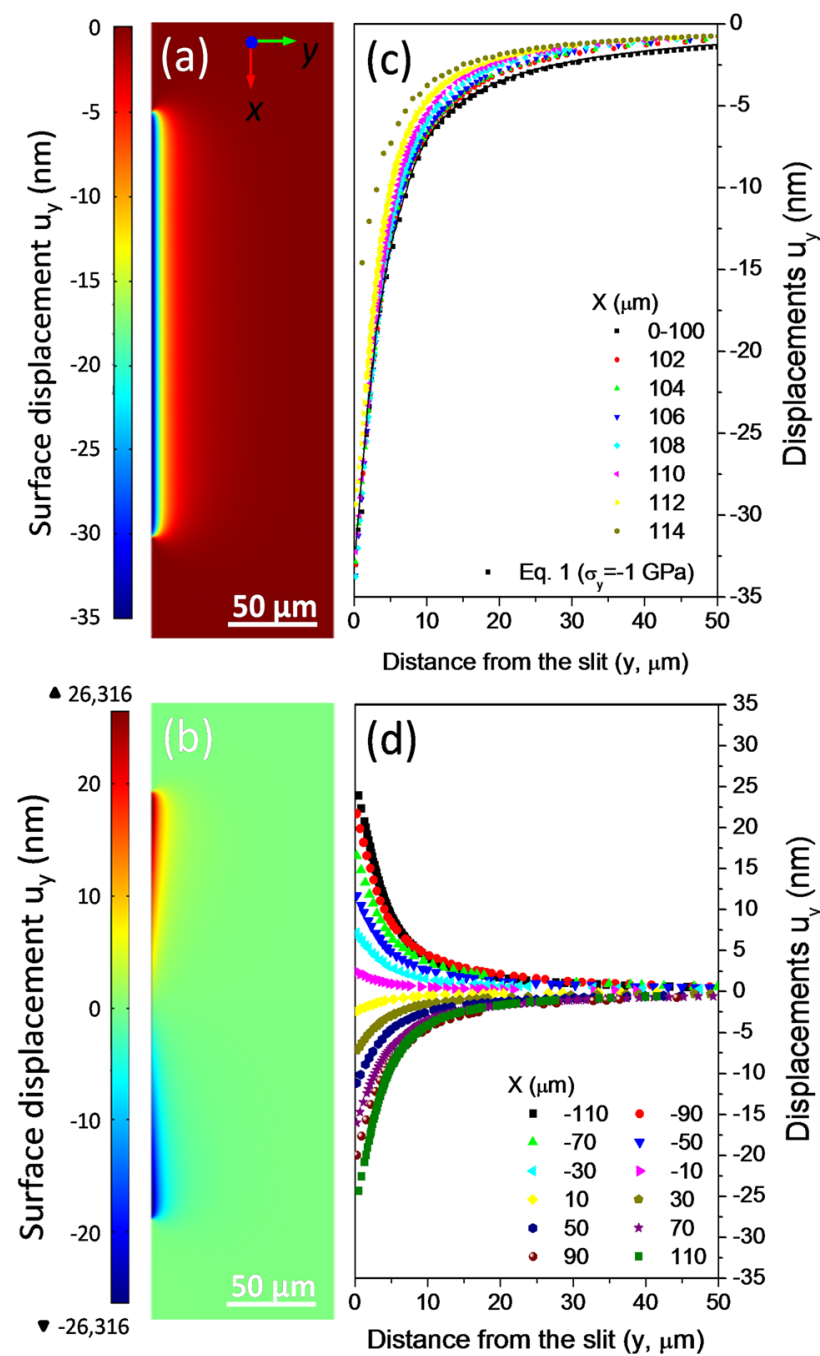

Fig. 7 Displacement fields in $y$ direction obtained by FEM in presence of uniaxial constant (a) and non-constant (b) stress fields. Constant value: $-1 \mathrm{GPa}$. Non-constant: linear variation from -0.82 to $+0.82 \mathrm{GPa}$ within the length of the slit $(230 \mu \mathrm{m})$, i.e., from compressive to tensile stress. The corresponding displacement profiles are shown in $\mathbf{c}$ and $\mathbf{d}$. Each color corresponds to a value of $x$, with $x=0$ representing the center of the slit. The profile obtained by the analytical solution is included for comparison (Color figure online)

profiles extracted from Fig. 7b. In this case, a transition from negative to positive displacements is observed, as a consequence of the tensile to compressive stress variation. Profiles do not cross each other, except of both at $x=110$ and $x=-110 \mu \mathrm{m}$, which cross the previous ones at $x=90$ and $x=-90$, respectively. This fact indicates that the proximity to the edge of the slit is operating there.

Similar to Fig. 6, Fig. 8 shows the comparison among the values obtained using Eq. 1 (symbols) for homogeneous (blue) and non-homogeneous (red) displacement fields. In both cases, the calculated results agree with the inputs (lines) around the center of the slit. For shorter slits,

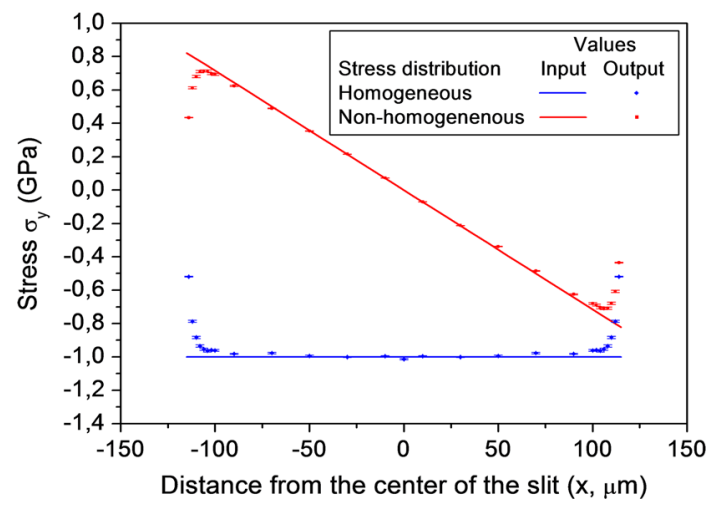

Fig. 8 Values of residual stress (output) at different distances from the center of the slit calculated using Eq. 1 on the profiles shown in Fig. $7 \mathrm{c}$ and d. Error bars represent the error of the fitting slope (see Fig. 1). Theoretical values of stresses used as input in the calculation are included for comparison

the deviation from the analytical elastic behavior occurred out of the central $60 \%$ of the slit length. However, for longer slits, the deviation starts much farther out of the central $90 \%$ of the slit length. This indicates that the use of longer slits, when possible, allows larger 'useful regions' where the analytical solution is still valid for getting the residual stress. Indeed, also based on the Saint-Venant principle that establishes the local nature of the effect of self-equilibrated external loads, we expect that the end effects for the current slits will not extend far beyond the maximum length scale of the depth of a couple of micrometers. That is so because at distances greater than the maximum linear dimensions of the region of load application, the stresses and deformations will be negligibly small. If $60 \%$ of 23 micrometers slit length can be used, one would expect that about $90 \%$ can be used if the slit becomes 10 times longer. The use of the longer slits should be also preferred because of another effect. As one moves further away from the slit (even at the slit center location), the influence of a finite slit length will start to reduce the displacements observed, assuming that the residual stress is large enough to generate displacements that can be resolved at these distances. This effect appears to be present in the example shown in Fig. 3 where, at distances greater than 6 micrometers from the slit, the observed displacements are smaller in magnitude than the predicted curves leading to larger errors. In this case, a slit length of only 12 microns was used. Because regions of 4-5 times the depth of the slit $\left(|y|<5 a_{\mathrm{f}}\right.$.) are considered for the displacement analysis at both sides of the slit, another criterion for slit length should be introduced: $L>10 a_{\mathrm{f}}$.

To verify the results displayed in Fig. $7 d$, where residual stresses change from compressive to tensile; a $200-\mu \mathrm{m}-$ thick steel strip under controlled 4-point bending was studied by the slit milling method in cross section. Figure 9 


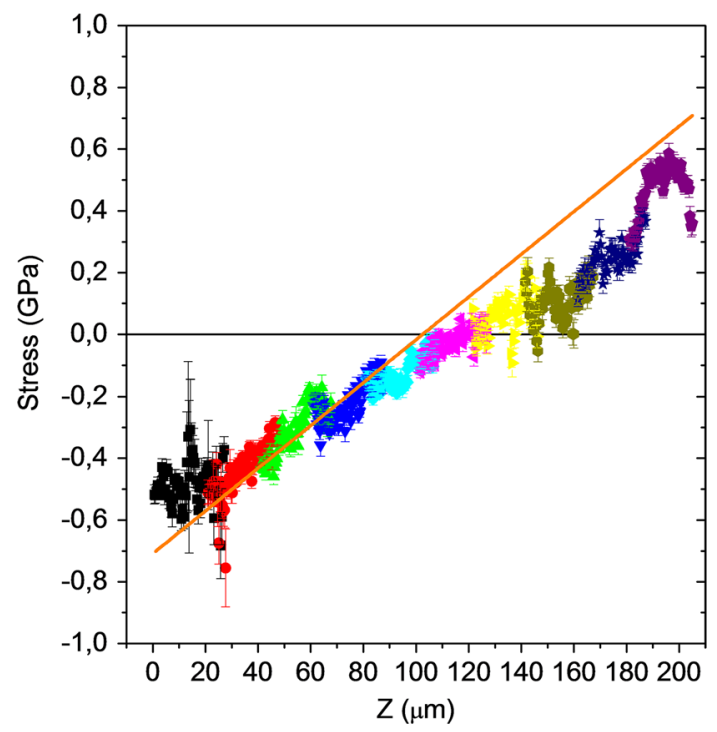

Fig. 9 Gradient of residual stress obtained by the slit milling method going from compressive (down part) to tensile (top part) on the 200- $\mu \mathrm{m}$-thick steel strip. Error bars represent the error of the fitting slope (see Fig. 1). Theoretical values calculated from measured strip curvature are included for comparison (see orange line) (Color figure online)

summarizes the values of residual stress when using the multiple fitting approach. As expected from a bending configuration, a non-symmetric behavior is observed, where the stress state varies through the cross section of the strip from negative values at the bottom part to positive values at the top part, i.e., from compressive to tensile stress. This behavior agrees quite well with the theoretical predictions from curvature (see orange line in Fig. 9), confirming the validity of the multiple fitting approach. Small deviations can be observed near to the edges of the slit, which are caused by the failure of the analytical solution, and the proximity to the end of the strip (top and bottom surfaces).

Thus, we can state that this method can be applied for any situation where a high degree of lateral resolution is required. For instance, measurements near inclusions in metals or in the vicinity of interfaces. Another possibility is the accurate evaluation of stress gradients. In that regard, Winiarsky and Witters [18] presented a method to measure internal stress gradients near the surface in depths of 1-2 $\mu \mathrm{m}$ by subsequent hole drilling. However, the approach presented in this work permits the accurate evaluation of this type of profile at long distances by milling only one slit in cross section (cf. Figs. 3b, 9). The final lateral resolution depends on the image resolution used in the SEM acquisition [34] and the facet size employed for the DIC procedure. In the examples shown in Fig. 3, adjacent points are separated by ca. $240 \mathrm{~nm}$, indicating the sub-micron resolution of this approach.
It is suggested that multiple fitting method should be used when one suspects a heterogeneous field of internal stresses. If the local stresses do not show any trend, the rather classical averaging method could be applied to increase the precision.

\section{Conclusions}

The paper presents a new approach in the evaluation of the so-called slit milling method that allows detailed information about local residual stress, particularly when stress gradients are present. It has been demonstrated to work for materials under tensile and compressive stresses.

It is shown that the range of applicability of the analytical solution is larger for longer slits. This is due to failure of the analytical solution near the edges, since it assumes an infinitely long slit. Therefore, protocols are preferred in which one long slit is milled instead of many smaller ones. Nevertheless, direct comparison with results from FEM simulations may aid to reveal the stress state from regions close to the slit edge. This approach can be used not only at a macro-level but also at a local micro-level for the evaluation of the stress fields around heterogeneous interfaces.

Acknowledgements This research was carried out under Project Number M61.7.10415 in the framework of the Research Program of the Materials Innovation Institute, M2i (www.m2i.nl).

Open Access This article is distributed under the terms of the Creative Commons Attribution License which permits any use, distribution, and reproduction in any medium, provided the original author(s) and the source are credited.

\section{References}

1. Totten GE, Howes MAH, Inoue T (2002) Handbook of residual stress and deformation of steel. ASM International, Materials Park, Ohio

2. Schajer GS (2013) Practical residual stress measurement methods. Wiley, Chichester

3. Withers PJ (2007) Residual stress and its role in failure. Rep Prog Phys 70:2211-2264. doi:10.1088/0034-4885/70/12/R04

4. Withers PJ, Bhadeshia HKDH (2001) Residual stress. Part 1measurement techniques. Mater Sci Technol 17:355-365. doi:10. 1179/026708301101509980

5. Prummer R, Pfeiffervollmar H (1983) A method for X-ray stressanalysis of thermochemically treated materials. Adv X-Ray Anal 26:225-231

6. Schajer GS, Prime MB (2006) Use of inverse solutions for residual stress measurements. J Eng Mater Technol 128:375-382. doi: $10.1115 / 1.2204952$

7. DeWald AT, Hill MR (2009) Eigenstrain-based model for prediction of laser peening residual stresses in arbitrary three-dimensional bodies. Part 1: model description. J Strain Anal Eng Des 44:1-11. doi:10.1243/03093247JSA417 
8. Schajer GS (2010) Relaxation methods for measuring residual stresses: techniques and opportunities. Exp Mech 50:1117-1127. doi:10.1007/s11340-010-9386-7

9. Martin CD, Christiansson RC (1991) Overcoring in highly stressed granite: comparison of USBM and modified CSIR devices. Rock Mech Rock Eng 24:207-235. doi:10.1007/BF01045032

10. Prime MB (1999) Residual stress measurement by successive extension of a slot: the crack compliance method. Appl Mech Rev 52:75-96. doi:10.1115/1.3098926

11. Cheng W (2007) Residual stress measurement and the slitting method. Springer, New York

12. Sabaté N, Vogel D, Gollhardt A et al (2006) Measurement of residual stresses in micromachined structures in a microregion. Appl Phys Lett 88:071910-0719113. doi:10.1063/1.2177357

13. Sabaté N, Vogel D, Keller J et al (2007) FIB-based technique for stress characterization on thin films for reliability purposes. Microelectron Eng 84:1783-1787. doi:10.1016/j.mee.2007.01.272

14. Sabate N, Vogel D, Gollhardt A et al (2007) Residual stress measurement on a MEMS structure with high-spatial resolution. J Microelectromechanical Syst 16:365-372. doi:10.1109/JMEMS.2006. 879701

15. Kang KJ, Yao N, He MY, Evans AG (2003) A method for in situ measurement of the residual stress in thin films by using the focused ion beam. Thin Solid Films 443:71-77. doi:10.1016/ S0040-6090(03)00946-5

16. McCarthy J, Pei Z, Becker M, Atteridge D (2000) FIB micromachined submicron thickness cantilevers for the study of thin film properties. Thin Solid Films 358:146-151. doi:10.1016/ S0040-6090(99)00680-X

17. Winiarski B, Withers PJ (2010) Mapping residual stress profiles at the micron scale using FIB micro-hole drilling. Appl Mech Mater 24-25:267-272. doi:10.4028/scientific.net/AMM.24-25. 267

18. Winiarski B, Withers PJ (2012) Micron-scale residual stress measurement by micro-hole drilling and digital image correlation. Exp Mech 52:417-428. doi:10.1007/s11340-011-9502-3

19. Sebastiani M, Eberl C, Bemporad E, Pharr GM (2011) Depthresolved residual stress analysis of thin coatings by a new FIBDIC method. Mater Sci Eng A 528:7901-7908. doi:10.1016/j. msea.2011.07.001

20. Sebastiani M, Bolelli G, Lusvarghi L et al (2012) High resolution residual stress measurement on amorphous and crystalline plasma-sprayed single-splats. Surf Coat Technol 206:4872-4880. doi:10.1016/j.surfcoat.2012.05.078

21. Zhu JG, Xie HM, Li YJ et al (2014) Interfacial residual stress analysis of thermal spray coatings by miniature ring-core cutting combined with DIC method. Exp Mech 54:127-136. doi:10.1007/ s11340-012-9640-2

22. Song X, Yeap KB, Zhu J et al (2012) Residual stress measurement in thin films at sub-micron scale using focused ion beam milling and imaging. Thin Solid Films 520:2073-2076. doi:10. 1016/j.tsf.2011.10.211

23. Krottenthaler M, Schmid C, Schaufler J et al (2013) A simple method for residual stress measurements in thin films by means of focused ion beam milling and digital image correlation. Surf Coat Technol 215:247-252. doi:10.1016/j.surfcoat.2012.08.095

24. Winiarski B, Langford RM, Tian J et al (2010) Mapping residual stress distributions at the micron scale in amorphous materials. Metall Mater Trans A 41:1743-1751. doi:10.1007/s11661-009$0127-4$

25. Korsunsky AM, Sebastiani M, Bemporad E (2009) Focused ion beam ring drilling for residual stress evaluation. Mater Lett 63:1961-1963. doi:10.1016/j.matlet.2009.06.020

26. Korsunsky AM, Sebastiani M, Bemporad E (2010) Residual stress evaluation at the micrometer scale: analysis of thin coatings by FIB milling and digital image correlation. Surf Coat Technol 205:2393-2403

27. Kahn-Jetter ZL, Chu TC (1990) Three-dimensional displacement measurements using digital image correlation and photogrammic analysis. Exp Mech 30:10-16

28. Sutton MA, Orteu JJ, Schreier HW (2009) Image correlation for shape, motion and deformation measurements-basic concepts, theory and applications. Springer, New York

29. Kang KJ, Darzens S, Choi GS (2004) Effect of geometry and materials on residual stress measurement in thin films by using the focused ion beam. J Eng Mater Technol 126:457-464. doi:10. $1115 / 1.1789965$

30. Sabaté N, Vogel D, Gollhardt A et al (2006) Measurement of residual stress by slot milling with focused ion-beam equipment. J Micromechanics Microeng 16:254-259. doi:10.1088/0960$1317 / 16 / 2 / 009$

31. Tada H, Paris CP, Irwin RG (2000) The stress analysis of cracks handbook. Edmunds, Bury St

32. Aramis (2004) GOM mbH (2004). Aramis v 5.3.0 user manual

33. Winiarski B, Schajer GS, Withers PJ (2012) Surface decoration for improving the accuracy of displacement measurements by digital image correlation in SEM. Exp Mech 52:793-804. doi:10. 1007/s11340-011-9568-y

34. Mansilla C, Ocelík V, De Hosson JTM (2014) A new methodology to analyze instabilities in SEM imaging. Microsc Microanal 20:1625-1637. doi:10.1017/S1431927614013282 